\title{
Computed Tomography Enterography: Better Luminal Distension with a Shorter Ingestion Time Protocol in an Indian Population
}

\author{
Ambika R. ${ }^{1} \quad$ Vidhya Rani Reddy ${ }^{1}$ \\ ${ }^{1}$ Department of Radiodiagnosis, Columbia Asia Referral Hospital, \\ Yeshwanthpur, Bengaluru, Karnataka, India
}

J Gastrointestinal Abdominal Radiol ISGAR 2018;1:33-40

\begin{abstract}
Address for correspondence Ambika R., MBBS, DMRD, DNB, Department of Radiology, KIMS, No. 16, Santrupti, Sampige Nagar Main, Rajeev Nagar Last Bus Stop, Vidya Nagar, Hubli 580031, Karnataka, India (e-mail: ambika13raja@gmail.com).
\end{abstract}

\begin{abstract}
Keywords

- computed tomogra-

phy enterography

- gut transit time

- oral contrast ingestion time

- polyethylene glycol

Aim This article prospectively evaluates the adequacy of bowel distension in computed tomography enterography (CTE) with a 45-minute contrast ingestion time and compares it with the most widely used protocol in the literature of 1 hour 20 minutes. Materials and Methods The CTE was performed in 42 consecutive patients divided into two groups $-A$ and B. Group A patients were instructed to drink $2 \mathrm{~L}$ of polyethylene glycol electrolyte solution over 1 hour 20 minutes. Group B patients were instructed to do the same over 45 minutes. At the end of contrast ingestion, plain and contrast CT abdomen was performed and CTE images were reviewed. Manual quantitative analysis of degree of small bowel distension was performed in the following manner: on coronal images, the abdominal cavity was divided into four quadrants: right upper, left upper, right lower, and left lower quadrants. The maximum small bowel lumen diameter (inner-to-inner wall) was measured in five different loops within each of the four quadrants. If four or more measurements in a quadrant $\geq 1.8 \mathrm{~cm}$ (considered "adequate luminal distension"), a score of 1 was assigned to that quadrant. If less than 4 measurements in the quadrant $>1.8 \mathrm{~cm}$, a score of 0 was assigned to that quadrant. The ensuing sum of scores from all four quadrants resulted in the distension grade for that CTE study (Grades 1-4).

Results There was a statistically significant difference in the degree of small bowel distension between the two groups with better distension seen in group $B(p<0.001)$. Conclusion Indians have a rapid gut transit time compared with Western populations. Hence, CTE contrast ingestion time protocols optimized in Western populations may not be suitable in Indians. The shorter 45 -minute ingestion protocol provided consistently better luminal distension in our population than the longer 1 hour 20-minute protocol described in the literature. To the best of our knowledge, there are no other studies comparing CTE ingestion time protocols in a given population.
\end{abstract}

\section{Introduction}

Despite advances in technology, the mesenteric small bowel continues to elude the reach of the endoscope. The barium meal follow through (BMFT) and the small bowel enteroclysis were the most commonly performed radiological examinations in suspected small bowel pathology. ${ }^{1,2}$ Recently, computed tomography (CT) and magnetic resonance imaging (MRI) of the abdomen after distension of the small bowel with neutral oral contrast, termed CT enterography (CTE) and MR enterography, have been increasingly advocated as an alternative to barium studies in view of the cross-sectional display of extraluminal structures, greater patient tolerance, and reduced procedural risks., ${ }^{3,4}$ The CTE with large volume oral contrast
License terms

(이 (1) $\Theta \circledast$ 
agent administered over a specified period of time provides adequate luminal distension and separation of small bowel loops, enabling high diagnostic accuracy for small bowel pathology. In CTE, detailed evaluation of the entire length of the small bowel is possible because of the uniform distension achieved by the ingestion of large volumes of contrast in a relatively short period of time. Thus, CTE allows simultaneous assessment of the lumen, wall thickness, and pattern of wall enhancement. CTE also eliminates the pitfalls associated with small bowel superimposition, allowing excellent depiction of mural and extraintestinal abnormalities. Multiple oral contrast agents including positive, negative, and neutral contrast agents have been tried to achieve small bowel distension on $\mathrm{CT}$ and it has been concluded that neutral oral agents are the most suitable for evaluation of small bowel pathology. ${ }^{5-9}$ In our study, we used polyethylene glycol (PEG) electrolyte solution to distend small bowel. Currently, the most commonly used CTE protocol is high volume (2 L) oral neutral contrast such as mannitol or PEG ingested over 1 hour 20 minutes..$^{3-5}$ Gut transit time can, however, vary with different populations. Indians have a shorter gut transit time as compared with Western population. Several factors such as age, gender, dietary habits, lifestyle, and biological differences may contribute for shorter gut transit time among Indians ${ }^{10-17}$ with mean stool frequency being higher in several Asian populations. For example, a stool frequency of thrice a week (range: 3-21 per week) is considered normal in a Western population, while Indians have a stool frequency of at least one stool per day. Two stools per day is considered as normal among Indians. ${ }^{16}$

Timing of contrast ingestion in enterography may therefore not be uniformly applicable to all populations. Given the rapid gut transit in Indians, we reduced the oral contrast ingestion time to 45 minutes and compared this with the standard protocol of 1 hour 20 minutes described in the literature. This study is therefore proposed to prospectively evaluate the luminal distension with shorter contrast ingestion time among Indians in comparison with the 1 hour 20 minutes ingestion time protocol.

\section{Materials and Methods}

\section{Patients and Control Subjects}

Our study is a prospective observational study with study population of 42 patients in the age group of 18 to 75 years referred to radiology for contrast-enhanced $\mathrm{CT}$ of the abdomen and pelvis to evaluate suspected small bowel pathology. All patients with a clinical suspicion of high grade, acute intestinal obstruction were excluded from the study. Other exclusion criteria included pregnancy, history of allergy to iodinated contrast, history of severe drug allergy, renal insufficiency with serum creatinine $>1.5 \mathrm{mg} / \mathrm{dL}$, and inability to ingest $>1 \mathrm{~L}$ of contrast. The patients were instructed to remain nil orally for solids for at least 4 hours prior to the start of CTE.

The CTE was performed in 42 consecutive patients divided into two equal groups-A and B. Group A patients were instructed to drink $2 \mathrm{~L}$ of neutral oral contrast (PEG electrolyte solution) over 1 hour 20 minutes. Group B patients were instructed to do the same over 45 minutes. At the end of contrast ingestion, routine $\mathrm{CT}$ abdomen was performed and the patient observed for 1 hour before leaving the hospital with an instruction to return to the hospital in case of serious side effects. Written informed consent was obtained from all patients. The study was conducted after Institutional Review Board and Ethics committee approval of the study protocol.

\section{Image Acquisition}

All scans were performed on a 64-slice CT scanner (Toshiba Aquilion). Using a pressure injector, $150 \mathrm{~mL}$ of intravenous (IV) Omnipaque ( $300 \mathrm{mg} / \mathrm{mL}$ ) was injected 4 to $4.5 \mathrm{~mL}$ / second. Administration of contrast was followed by a flush of $40 \mathrm{~mL}$ normal saline at the same injection rate. A dual-phase CT scan was performed after an unenhanced CT scan. Bolus tracking method was used for acquisition of arterial and enteric phases; the trigger was placed on the descending thoracic aorta, arterial phase acquired after a delay of 15 to 20 seconds, and enteric phase after a delay of 45 seconds postthreshold achievement in the lower thoracic aorta.

\section{Postprocessing}

Images were acquired in the axial plane in a cephalocaudal direction, from the hepatic dome to the symphysis pubis, during one breath hold. Raw data were generated in axial planes with 3-mm thickness, later reconstructed in coronal and sagittal planes with a section thickness of $3 \mathrm{~mm}$ and reconstruction interval of 1 to $1.5 \mathrm{~mm}$. Images were transferred to picture archiving and communication system for review. In addition, the $0.625-\mathrm{mm}$ raw data were transferred to the workstation for three-dimensional volume rendering and maximum-intensity-projection displays.

\section{Image Analysis}

Manual quantitative analysis of the degree of small bowel distension was performed in the following manner: on coronal images, the abdominal cavity was divided into four quadrants: right upper, left upper, right lower, and left lower quadrants. The maximum small bowel lumen diameter (inner-to-inner wall) was measured in five different loops within each of the four quadrants. If 4 or more measurements in a quadrant $\geq 1.8 \mathrm{~cm}$ (considered "adequate luminal distension"), a score of 1 was assigned to that quadrant. If less than 4 measurements in the quadrant $>1.8 \mathrm{~cm}$, a score of 0 was assigned to that quadrant. The ensuing sum of scores from all four quadrants resulted in the distension grade for that CTE study (Grades 1-4).

If each of the four quadrants scored 1 , then distension grade for that CTE study is Grade 4. Similarly, if sum of all the four quadrants is 3 , then the distension grade for that CTE study would be Grade 3, if sum of all four quadrants is 2 , then the distension grade would be Grade 2, and if sum of all four quadrants is 1 , then the distension grade would be Grade 1 .

\begin{tabular}{|l|l|}
\hline $\begin{array}{l}\text { Sum of all } \\
\mathbf{4} \text { quadrants }\end{array}$ & $\begin{array}{l}\text { Distension grade for } \\
\text { the CTE study }\end{array}$ \\
\hline $4 / 4$ & Grade 4 \\
\hline $3 / 3$ & Grade 3 \\
\hline $2 / 4$ & Grade 2 \\
\hline $1 / 4$ & Grade 1 \\
\hline
\end{tabular}



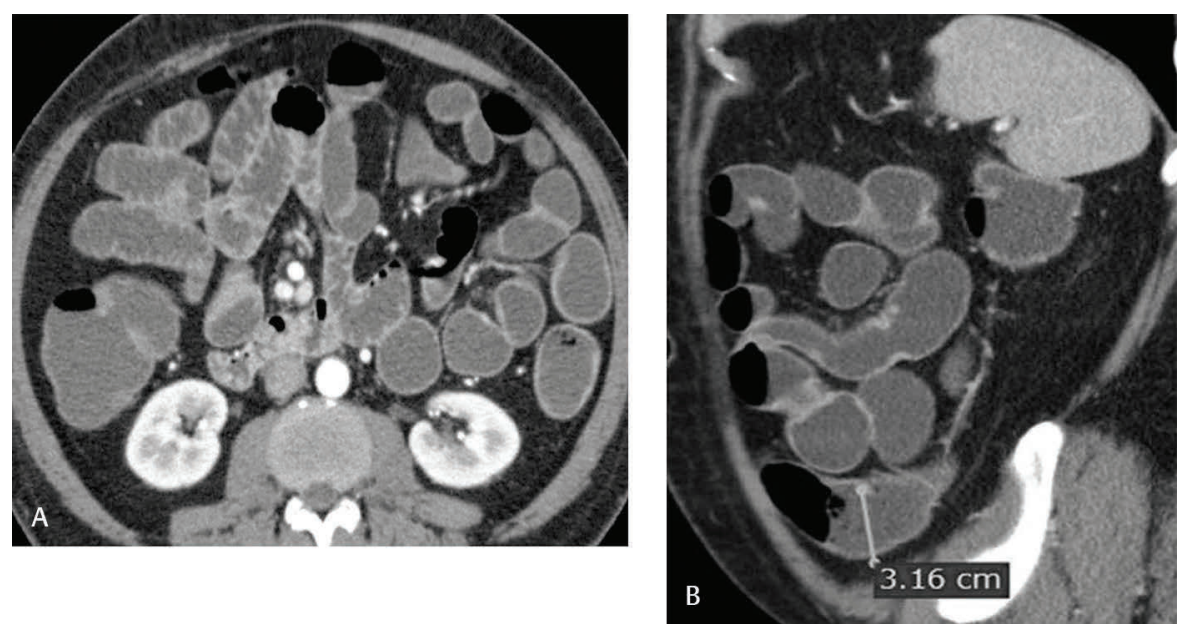

Fig. 1 Axial (A) and sagittal (B) reconstructed computed tomography (CT) enterography images in enteric phase of a 30-year-old female patient showing Grade 4 distension score.

Grades 4 and 3 were considered to have optimal bowel distension (-Figs. 1-4), while Grades 2 and 1 (-Figs. 5-9) were considered to have poor bowel distension. None of our patients had Grade 1 distension.

Data were tabulated and analyzed using statistical methods.

\section{Statistical Analysis}

Descriptive and inferential statistical analyses were done on the data collected and tabulated. Results on continuous measurements are presented as mean \pm standard deviation (min-max) and results on categorical measurements presented in number (\%). Chi-square/Fisher's exact test was used to find the significance of study parameters on categorical scale between two or more groups.

Statistical software: The Statistical software, namely SAS 9.2, SPSS 15.0, Stata 10.1, MedCalc 9.0.1, Systat 12.0, and $\mathrm{R}$ environment ver.2.11.1 were used for the analysis of the data and Microsoft Word and Excel were used to generate graphs, tables, etc.

\section{Results}

Both the protocols were well tolerated by the patients, without any discomfort; none of the 42 patients who ingested 2 L PEG solution reported any major side effects. Forty-two patients ingested $2 \mathrm{~L}$ of oral contrast in the stipulated time without significant nausea or abdominal discomfort. There was a statistically significant difference in the degree of small bowel distension between the two groups with better distension seen in group B with shorter contrast ingestion time protocol $(p<0.001)$. Grade 4 distension was achieved in only $9.5 \%$ of group A subjects with the longer 1 hour 20-minute protocol as against $42.9 \%$ in group B. Grade 3 distension was achieved only in $33.3 \%$ of group A subjects as against $42.9 \%$ of group B. Overall, adequate and more uniform distension of small bowel was achieved with the group B 45-minute ingestion protocol compared with the group A 1 hour 20-minute protocol ( - Table 1, - Fig. 10).

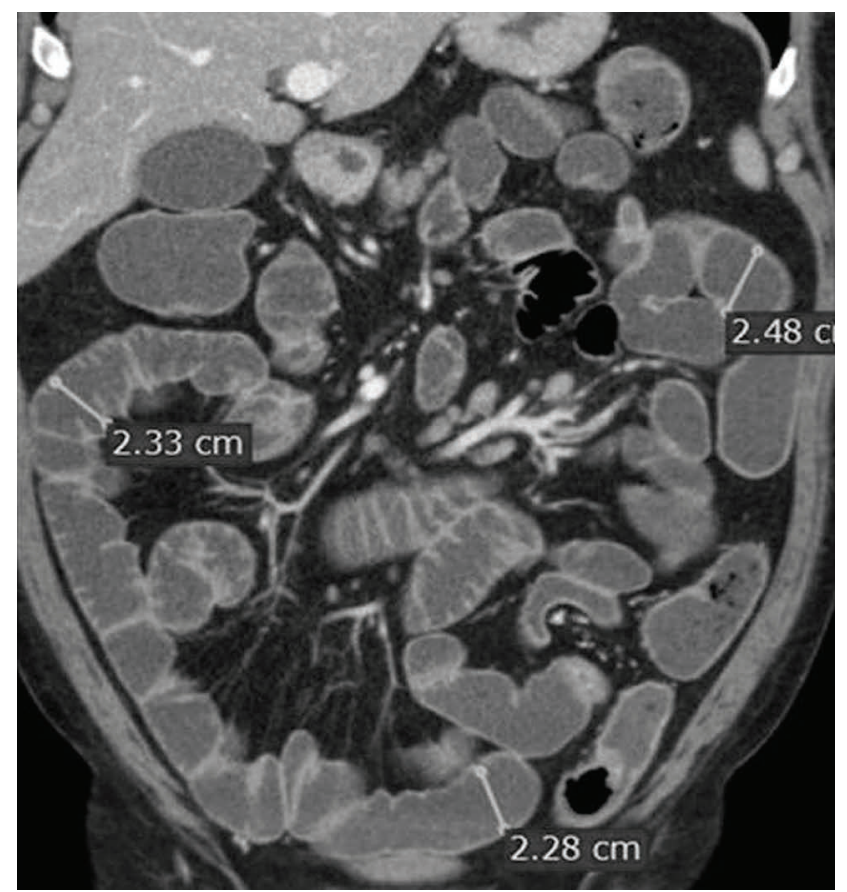

Fig. 2 A 24-year-old male patient with normal computed tomography $(\mathrm{CT})$ enterography study. Coronal reformation through abdomen in enteric phase demonstrating Grade 3 distension score.

Incidence of small bowel pathology was analyzed in two groups separately. $47.6 \%$ patients of group A had small bowel pathology while $23.8 \%$ of patients in group B had small bowel pathology. Thus, the bias associated with small bowel pathology induced shorter transit times was eliminated (-Tables 1 and 2, -Figs. 1-11).

\section{Discussion}

\section{Computed Tomography Enterography}

Despite advances in technology, the mesenteric small bowel continues to elude the reach of the endoscope and provides the biggest challenge in bowel imaging. This is mainly due to the length, redundancy, overlap, and small caliber of the 

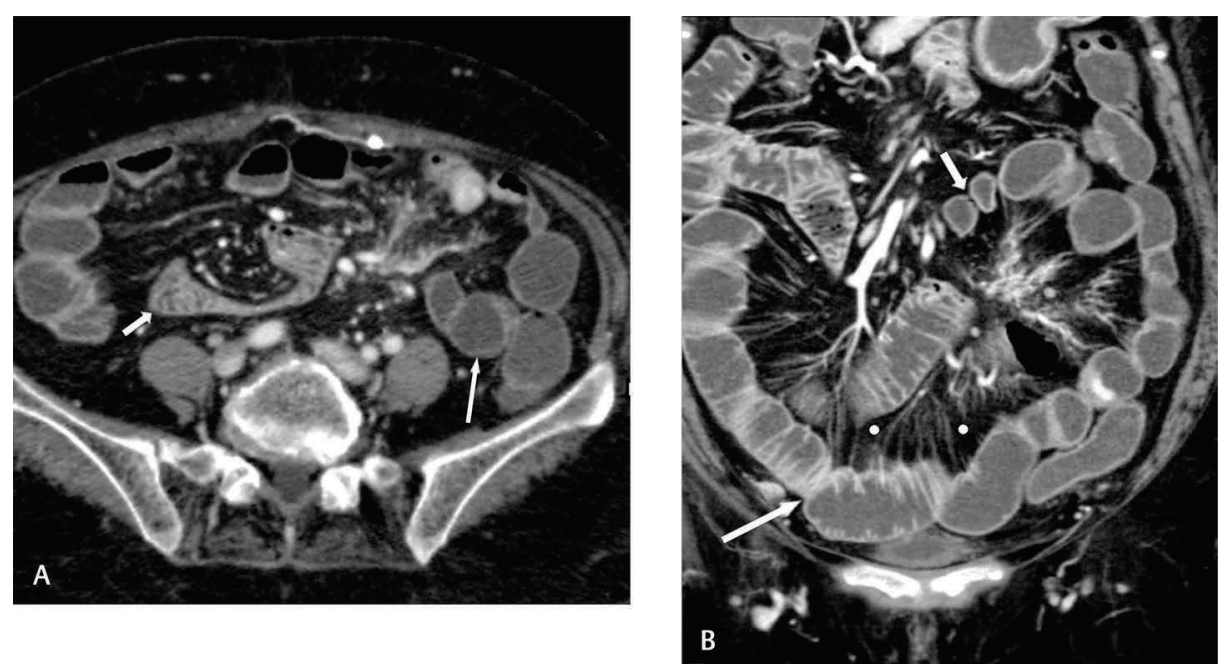

Fig. 3 A 73-year-old female patient, proven case of Crohn's disease with Grade 3 distension score computed tomography (CT) enterography study. Axial (A) and coronal reformation (B) through abdomen in enteric phase demonstrating both poorly distended (short arrows) loops and well distended (long arrows) small bowel loops. The image also demonstrates engorged vasa recta (positive comb's sign) (white circle).

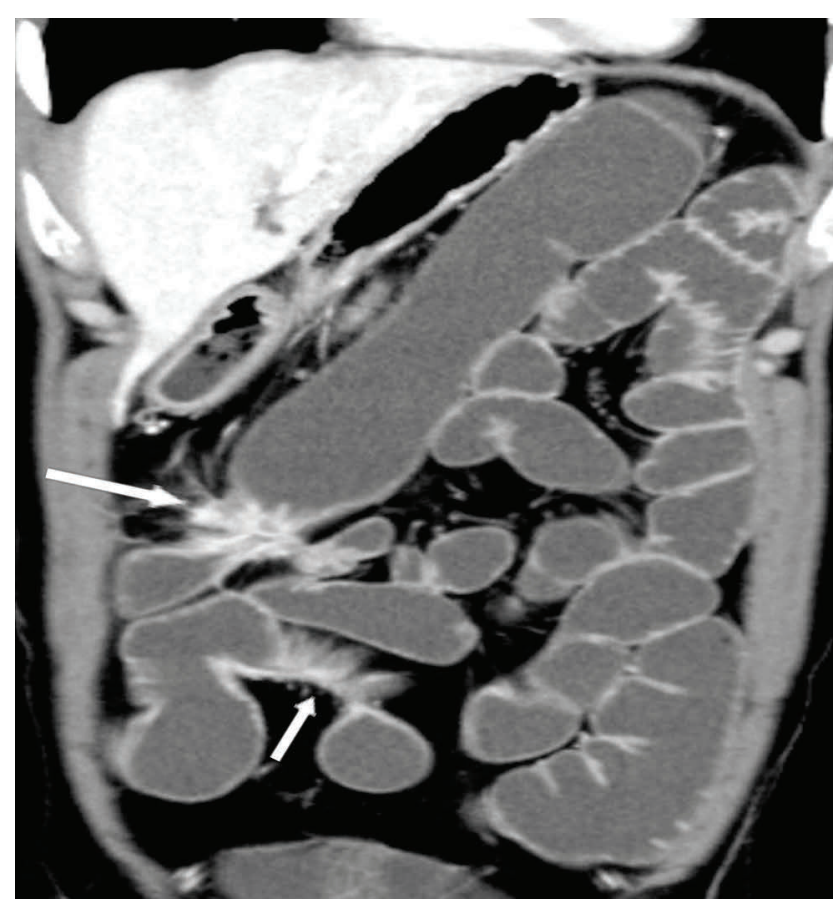

Fig. 4 Computed tomography (CT) enterography study (with Grade 4 distension score) of a 36-year-old male patient, known case of Crohn's disease presented with recurrent pain abdomen 1 year postresection. Coronal reformation in enteric phase shows short segment stricture with wall thickening and marked enhancement suggesting recurrence at the anastomotic site (long arrow). The short arrow points to another similar segment (just inferior) with wall thickening, enhancement, and pseudosacculations (short arrow).

small bowel. Because of the overlapping nature of bowel loops in the pelvis, inadequate and nonuniform distension of loops, lengthy procedure time, barium-related complications, and operator dependence associated with barium studies, cross-sectional imaging studies are becoming more popular for evaluation of small bowel pathology. Cross-sectional imaging of small bowel includes CT and MRI with oral and IV contrast agents. These studies not only prevent obscuration

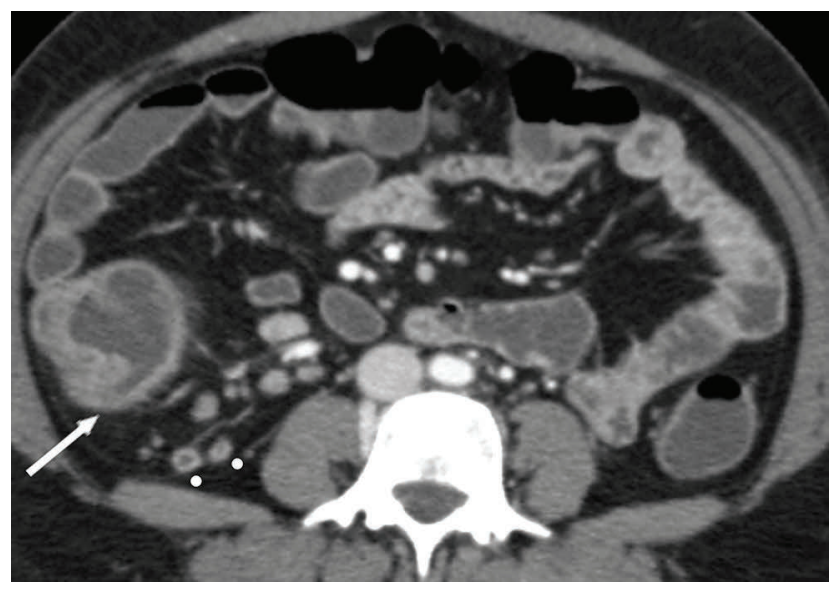

Fig. 5 A 26-year-old female patient, proven case of abdominal tuberculosis. Axial computed tomography (CT) enterography image in enteric phase demonstrating Grade 2 distension score with collapsed small bowel loops. The image shows asymmetrical wall thickening involving ileocecal junction (arrow) with multiple necrotic mesenteric lymph nodes in right lower quadrant.

of small bowel loops by superimposition, but also depict mural and extraintestinal complications of the disease. They provide better depiction of small sinus tracts, abscesses, fistulas, and ulcerations especially when combined with oral contrast agents either via enterography or enteroclysis. ${ }^{1,2}$

The CT abdomen after distension of the small bowel with neutral oral contrast, termed CTE, has been increasingly advocated as an alternative to barium studies. CTE is a simple, noninvasive radiological investigation for evaluating small bowel and can be used as the primary investigation in suspected small bowel pathology. Irrespective of the CT technique used, adequate bowel distension is mandatory since mural thickening is the hallmark of small bowel disease. Complete evaluation of the small bowel is achieved with the addition of IV contrast and a large volume of neutral contrast to distend the intestinal lumen, allowing assessment of the lumen, thickness, and pattern of enhancement of the 
small intestinal wall. The CTE with large volume of neutral oral contrast agent provides adequate luminal distension and separation of small bowel loops, thus accounting for a high diagnostic accuracy for small bowel pathology. Currently, CTE is commonly performed using high-volume (2 L) oral neutral contrast agents such as mannitol or PEG ingested over 60 to 90 minutes followed by an IV contrast. Many authors have reported CTE to be highly sensitive and specific in the diagnosis and characterization of various small bowel pathologies. Solem et al performed a comparison study

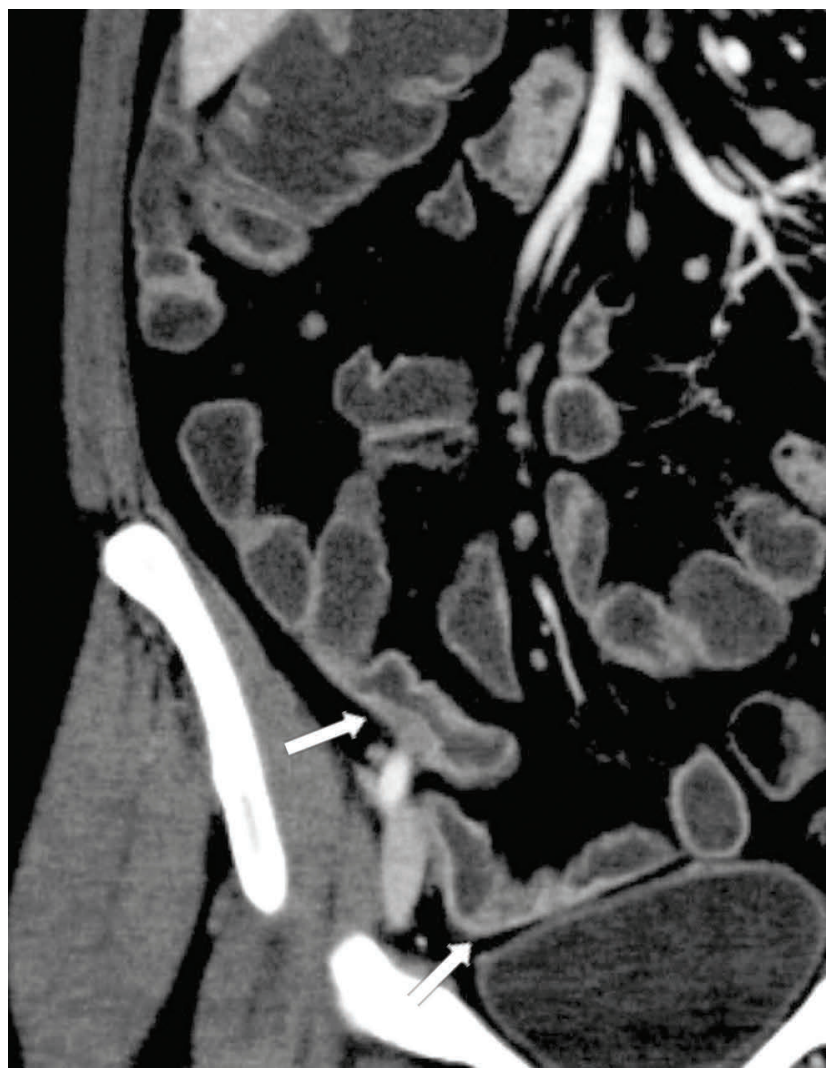

Fig. 6 A 30-year-old female patient, biopsy-proven case of Crohn's disease demonstrating Grade 2 distension score computed tomography (CT) enterography study. Coronal reformation through the abdomen in the enteric phase shows skip lesions in the ileal loops located in the right lower quadrant (arrows). between CTE, ileocolonoscopy, capsule endoscopy, and BMFT on 41 patients with Crohn's disease and concluded that the sensitivity of CTE was equal to that of capsule endoscopy (83\%) and more than that of ileocolonoscopy (74\%) and BMFT (65\%). In this study, specificity of CTE (82\%) was found to be less than that of ileocolonoscopy (100\%) but more than that of capsule endoscopy (53\%) and BMFT (94\%). ${ }^{18}$ In 2011, Minordi et al compared CTE and CT enteroclysis in 145 patients. Seventy patients underwent CT enteroclysis after jejunal intubation and infusion of methylcellulose, and 75 patients underwent CTE after orally ingesting $2 \mathrm{~L}$ of PEG solution over 45 minutes. CTE showed findings of Crohn's disease as well as CT enteroclysis, although CT enteroclysis gave better bowel distension, especially in the jejunum, and had a higher specificity than CTE. ${ }^{19}$

A study performed in 2011 by Huprich et al on 22 patients demonstrated that multiphasic CTE was more than twice as sensitive as capsule endoscopy for finding the source of obscure gastrointestinal (GI) bleeding ( 88 vs. $38 \%$ ). ${ }^{20}$ In India, Sodhi et al in 2012 performed CTE on 35 patients with occult GI bleed-15 had positive findings that were confirmed on

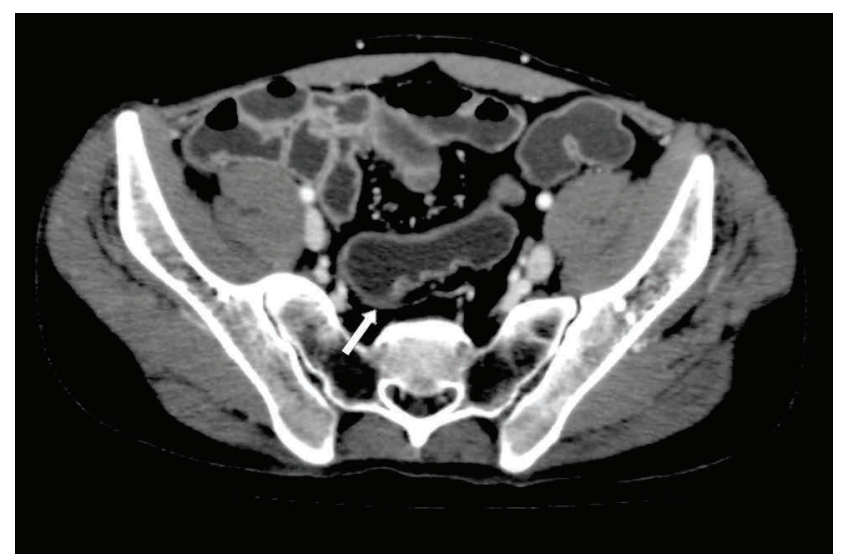

Fig. 7 Computed tomography (CT) enterography study with Grade 2 distension score in a 35-year-old male patient, known case of Crohn's disease presented with recurrent pain abdomen. Axial CT enterography image in enteric phase demonstrating pseudosacculations along the antimesenteric border of sigmoid colon and shortening of the mesenteric border (arrow).
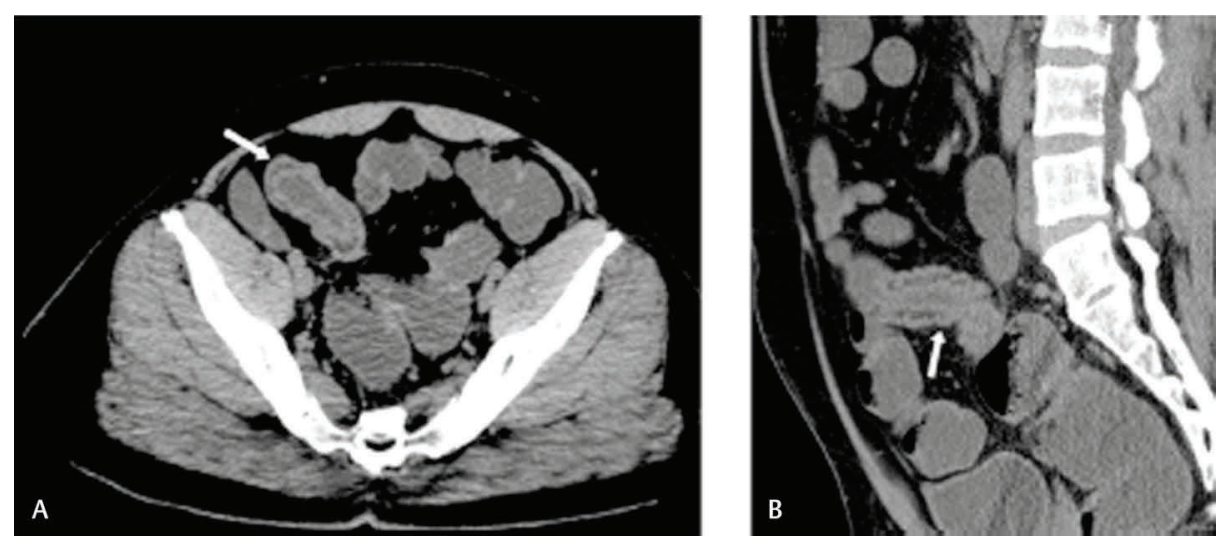

Fig. 8 A 37-year-old male patient, biopsy-proven case of Crohn's disease. Axial (A) and sagittal (B) reformatted plain computed tomography (CT) enterography image with Grade 2 bowel distension. Stratified wall thickening (arrows) is seen in the affected distal ileal segment. 

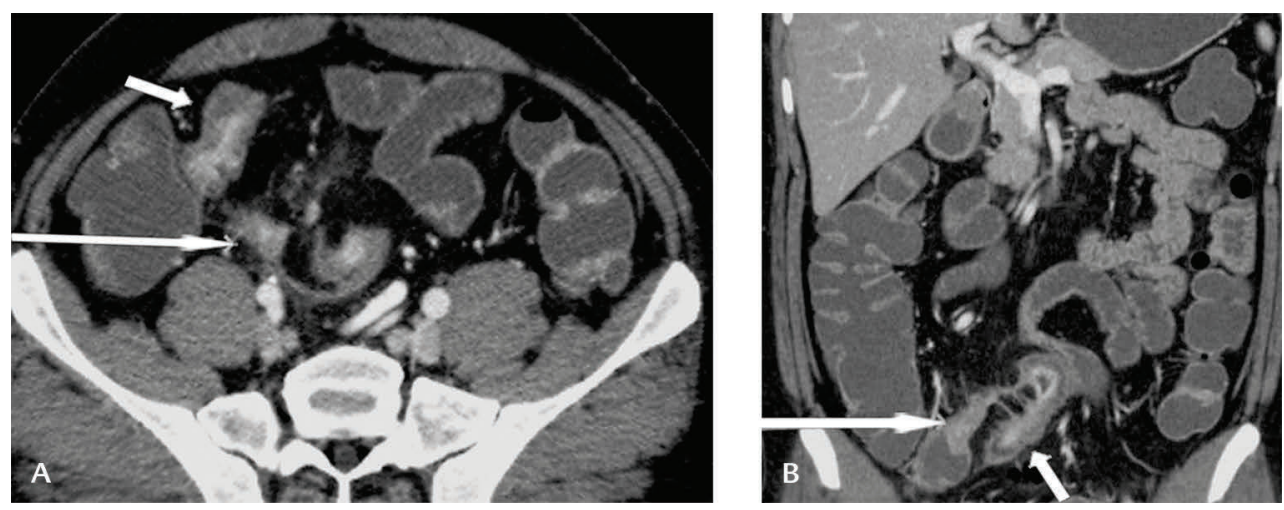

Fig. 9 Computed tomography (CT) enterography study of a 37-year-old male patient, suspected case of Crohn's disease demonstrating poor/ Grade 2 distension score. Axial (A) and reconstructed coronal (B) CT enterography image in the enteric phase shows thickening of the ileocecal junction, terminal ileal loop with engorged vasa recta/positive Comb's sign (short arrows). The image also demonstrates enhancing appendix (long arrows). Histopathological examination (HPE) revealed Crohn's disease.

Table 1 Comparison of small bowel diameter in two groups with different contrast ingestion times: Group A (1 hour 20 minutes) and group $B$ (45 minutes)

\begin{tabular}{|l|l|l|l|}
\hline & Group A & Group B & $p$-Value \\
\hline Right upper quadrant & $1.92 \pm 0.26$ & $2.14 \pm 0.28$ & $0.013^{*}$ \\
\hline Left upper quadrant & $2.76 \pm 3.74$ & $2.25 \pm 0.31$ & 0.541 \\
\hline Right lower quadrant & $2.04 \pm 0.33$ & $2.20 \pm 0.34$ & 0.112 \\
\hline Left lower quadrant & $1.95 \pm 0.26$ & $2.16 \pm 0.32$ & $0.021^{*}$ \\
\hline
\end{tabular}

*Statistically significant $(p<0.05)$.

Table 2 Grade/Distension scores

\begin{tabular}{|l|l|l|l|l|}
\hline Grade/distension score & $\begin{array}{l}\text { Group A } \\
\text { (number) }\end{array}$ & $\begin{array}{l}\text { Group A } \\
\text { Percentage (\%) }\end{array}$ & $\begin{array}{l}\text { Group B } \\
\text { (number) }\end{array}$ & $\begin{array}{l}\text { Group B } \\
\text { Percentage (\%) }\end{array}$ \\
\hline Grade 1 & 0 & 0.0 & 0 & 0.0 \\
\hline Grade 2 & 12 & 57.1 & 3 & 14.3 \\
\hline Grade 3 & 7 & 33.3 & 9 & 42.9 \\
\hline Grade 4 & 2 & 9.5 & 9 & 42.9 \\
\hline Total & 21 & 100 & 21 & 100 \\
\hline
\end{tabular}

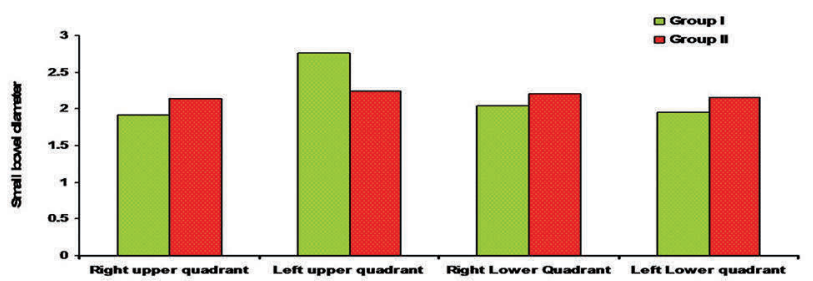

Fig. 10 Comparison of small bowel diameter in two groups.

exploratory laparotomy. They concluded that CTE was a useful investigation tool in the evaluation of both occult and overt GI bleeding. ${ }^{21}$

\section{Polyethylene Glycol as Oral Neutral Contrast Agent}

Multiple oral contrast agents have tried to achieve small bowel distension on CT including positive, negative, and neutral contrast agents. Neutral oral agents have been found to be the most suitable for the evaluation of small bowel

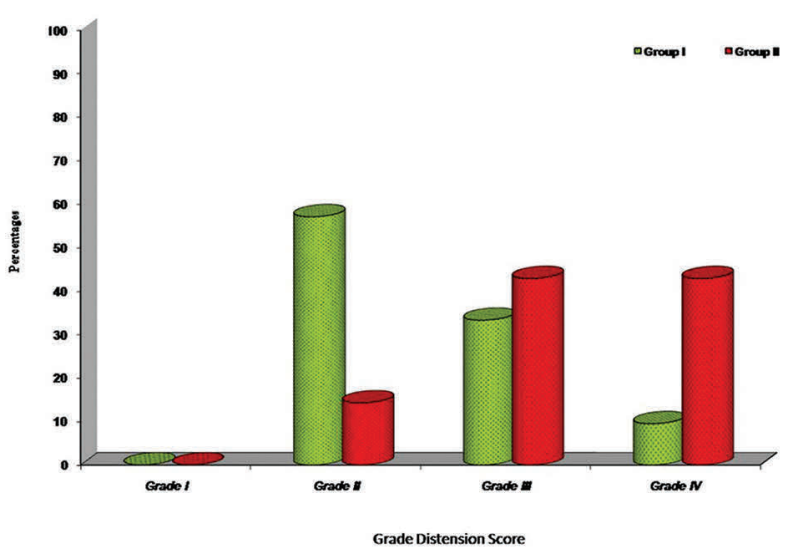

Fig. 11 Grade/distension score-comparison between two groups: Group I (A) and group II (B).

pathology. ${ }^{5,6,7}$ PEG electrolyte solution has been shown to distend small bowel better than water or methyl cellulose solution as well as low-density barium. ${ }^{8,9}$ The neutral oral 
contrast agent in conjunction with IV contrast results in the depiction of the lumen as well as the thickness and enhancement pattern of the wall. PEG electrolyte solution is the best known neutral contrast used widely as a colonoscopy preparatory agent. It is easily available, less expensive, palatable, and has no known serious side effects. ${ }^{5-9}$ CTE with PEG solution was performed by Minordi et al and found to be $93 \%$ sensitive and $94 \%$ specific compared with CT enteroclysis that had a sensitivity of $94 \%$ and specificity of $100 \% .{ }^{19}$ In our study, PEG electrolyte was well tolerated in concordance with the studies conducted by Solem et al and Minordi et al. ${ }^{18,19}$

\section{Oral Contrast Ingestion Time in Computed Tomography Enterography}

It is a well-known fact that gut transit time varies widely with different populations, races, and ethnicity. Age, gender, dietary habits, degree of physical activity, and biological factors are some factors influencing the gut transit time. Hence, GI protocols used in one population may not be globally applied on all populations. ${ }^{10-17}$ There is paucity of literature on normal range of gut transit time in healthy Indian subjects and ideal or standard oral contrast ingestion time to be used among Indian population. However, Indians are found to have a rapid gut transit time compared with the Western population. The stool frequency is considerably higher among the Asian population. Mean stool frequency is higher in several Asian populations. For example, a stool frequency of thrice a week (range: 3-21 per week) is considered normal in a Western population while Indians have a stool frequency of at least one stool per day. Two stools per day is considered as normal among Indians..$^{16}$ The most commonly used CTE protocol in the West is high-volume $(2 \mathrm{~L})$ oral neutral contrast agents such as mannitol or PEG ingested over 1 hour 20 minutes. In view of faster gut transit time, we postulated that Indian populations may need a shorter contrast ingestion time. In our study, we decreased the oral contrast ingestion time to 45 minutes and compared this with the standard protocol of 1 hour 20 minutes described in the literature. We found that ingestion of oral contrast over a period of 1 hour 20 minutes provided suboptimal distension of the small bowel, particularly the jejunum with more uniform distension of the colon. This is likely due to rapid gut transit time among Indians. Administration of the oral contrast agent over a period of 45 minutes resulted in improved distension of the jejunum and a more uniform distension of the small bowel. A similar protocol of a 45-minute oral contrast ingestion time was recommended for optimal bowel distension by Ilangovan et $\mathrm{al}^{4}$, where patients were instructed to drink $2 \mathrm{~L}$ of $2.5 \%$ mannitol solution over 45 minutes. Minordi et al in 2011also used a similar protocol with 2 L of PEG solution administered over 45 minutes and achieved optimal bowel distension. Their study concluded that results obtained with PEG CTE using the 45-minute oral contrast ingestion protocol for demonstrating findings of Crohn's disease was comparable with that of CT enteroclysis. ${ }^{19}$

Limitations: We addressed bias due to shortening of gut transit because of small bowel disease, by comparing the occurrence of small bowel pathology between the two groups. We found that the shorter ingestion protocol group had fewer patients with small bowel pathology (24 vs. 48\%), thus eliminating this potential bias. However, our study had other limitations: our sample size of 42 was relatively small and we did not match our patients for age, gender, or diet in the two groups; intrinsic differences in gut transit time between the two groups were therefore not corrected.

\section{Conclusion}

Gut transit time can vary amongst different ethnicities. CTE contrast ingestion protocols need to be optimized for specific populations. Populations with faster gut transit time need shorter oral contrast ingestion time. CTE contrast ingestion protocols optimized in one population may not be suitable in another population. We found statistically significant improvement in small bowel distension with the shorter 45-minute ingestion CTE protocol compared with the 1 hour 20-minute protocol recommended by many authors. Ours is the first study to compare two contrast ingestion time protocols in an Indian population. To the best of our knowledge, there are no other studies comparing CTE oral contrast ingestion time protocols in a given population. Further studies with a larger sample size are recommended to investigate optimum oral contrast ingestion protocols for CTE among different populations.

\section{Funding \\ None. \\ Conflict of Interest \\ None.}

\section{References}

1 Gore R, Levine M. Barium examinations of small intestine. Textbook of Gastrointestinal Radiology. 3rd ed. Philadelphia, PA: Saunders; 2007:735-897

2 Wold PB, Fletcher JG, Johnson CD, Sandborn WJ. Assessment of small bowel Crohn disease: noninvasive peroral CT enterography compared with other imaging methods and endoscopy--feasibility study. Radiology 2003;229(1):275-281

3 Paulsen SR, Huprich JE, Fletcher JG, et al. CT enterography as a diagnostic tool in evaluating small bowel disorders: review of clinical experience with over 700 cases. Radiographics 2006;26(3):641-657, discussion 657-662

4 Ilangovan R, Burling D, George A, Gupta A, Marshall M, Taylor SA. CT enterography: review of technique and practical tips. Br J Radiol 2012;85(1015):876-886

5 Macari M, Balthazar EJ. CT of bowel wall thickening: significance and pitfalls of interpretation. Am J Roentgenol 2001;176(5):1105-1116

6 Upegui J Daniel, Mendoza B Oscar D, Segura C Wilber O, Heredia S Fabian M, Galvis $\mathrm{R}$ German, Fuentes Jorge E. Use of CT enterography forsmall bowel pathology: experience and findings in 90 patients. Revisit colombiana de Radiologia 2010;21(1):2818-2825

7 Megibow AJ, Babb JS, Hecht EM, et al. Evaluation of bowel distention and bowel wall appearance by using neutral oral contrast agent for multi-detector row CT. Radiology 2006;238(1):87-95

8 Prakashini K, Kakkar C, Sambhaji C, Shetty CM, Rao VR. Quantitative and qualitative bowel analysis using mannitol, water and iodine-based endoluminal contrast agent on 64-row detector CT. Indian J Radiol Imaging 2013;23(4):373-378 
9 Young BM, Fletcher JG, Booya F, et al. Head-to-head comparison of oral contrast agents for cross-sectional enterography: small bowel distention, timing, and side effects. J Comput Assist Tomogr 2008;32(1):32-38

10 Ghoshal UC, Gupta D, Kumar A, Misra A. Colonic transit study by radio-opaque markers to investigate constipation: validation of a new protocol for a population with rapid gut transit. Natl Med J India 2007;20(5):225-229

11 Jayanthi V, Chacko A, Gani IK, Mathan VI. Intestinal transit in healthy southern Indian subjects and in patients with tropical sprue. Gut 1989;30(1):35-38

12 Sadik R, Abrahamsson H, Stotzer PO. Gender differences in gut transit shown with a newly developed radiological procedure. Scand J Gastroenterol 2003;38(1):36-42

13 Metcalf AM, Phillips SF, Zinsmeister AR, MacCarty RL, Beart RW, Wolff BG. Simplified assessment of segmental colonic transit. Gastroenterology 1987;92(1):40-47

14 Arhan P, Devroede G, Jehannin B, et al. Segmental colonic transit time. Dis Colon Rectum 1981;24(8):625-629

15 Pai CG, Kurian G. A modified radiographic method for estimating segmental colonic transit time in subjects with rapid gut transit. Indian J Med Res 1999;110:22-26
16 Panigrahi MK, Kar SK, Singh SP, Ghoshal UC. Defecation frequency and stool form in a coastal eastern Indian population. J Neurogastroenterol Motil 2013;19(3):374-380

17 Thankappan KR, Narendranathan M. Normal bowel habits: results of a study in Kerala. Indian J Gastroenterol 1992;11(suppl 1):A3

18 Solem CA, Loftus EV Jr, Fletcher JG, et al. Small-bowel imaging in Crohn's disease: a prospective, blinded, 4-way comparison trial. Gastrointest Endosc 2008;68(2):255-266

19 Minordi LM, Vecchioli A, Mirk P, Bonomo L. CT enterography with polyethylene glycol solution vs CT enteroclysis in small bowel disease. Br J Radiol 2011;84(998):112-119

20 Huprich JE, Fletcher JG, Filder JL, Liano E. Obscure GI bleeding: the role of multiphase CT enterography. Appl Radiol 2011;40(12):16-20

21 Sodhi JS, Zargar SA, Rashid W, et al. 64-section multiphase CT enterography as a diagnostic tool in the evaluation of obscure gastrointestinal bleeding. Indian $\mathrm{J}$ Gastroenterol 2012;31(2):61-68 